\title{
Scientific imperatives, clinical implications, and theoretical underpinnings for the investigation of the relationship between genetic variables and patient-reported quality-of-life outcomes
}

\author{
Mirjam A. G. Sprangers · Jeff A. Sloan • Andrea Barsevick • \\ Cynthia Chauhan · Amylou C. Dueck · Hein Raat • \\ Quiling Shi · Cornelis J. F. Van Noorden · The GENEQOL Consortium
}

Accepted: 25 September 2010/Published online: 14 October 2010

(C) The Author(s) 2010. This article is published with open access at Springerlink.com

\begin{abstract}
Objectives There is emerging evidence for a genetic basis of patient-reported quality-of-life (QOL) outcomes that can ultimately be incorporated into clinical research and practice. Objectives are (1) to provide arguments for the timeliness of investigating the genetic basis of QOL given the scientific advances in genetics and patient-reported QOL research; (2) to describe the clinical implications of
\end{abstract}

M. A. G. Sprangers $(\square)$

Department of Medical Psychology/J3-211, Academic Medical Center, University of Amsterdam, Meibergdreef 15,

1105 AZ Amsterdam, The Netherlands

e-mail: m.a.sprangers@amc.uva.nl

\section{J. A. Sloan}

Department of Health Sciences Research, Mayo Clinic,

Rochester, MN, USA

A. Barsevick

Cancer Prevention and Control Program, Fox Chase Cancer

Center, Philadelphia, PA, USA

C. Chauhan

Cancer Advocacy, Wichita, KS, USA

A. C. Dueck

Section of Biostatistics, Mayo Clinic, Scottsdale, AZ, USA

H. Raat

Department of Public Health, Erasmus Medical Center, University Medical Center Rotterdam, Rotterdam,

The Netherlands

Q. Shi

Department of Symptom Research, The University of Texas M. D. Anderson Cancer Center, Houston, TX, US

C. J. F. Van Noorden

Department of Cell Biology and Histology, Academic Medical Center, University of Amsterdam, Amsterdam, The Netherlands such investigations; (3) to present a theoretical foundation for investigating the genetic underpinnings of QOL; and (4) to describe a series of papers resulting from the GENEQOL Consortium that was established to move this work forward.

Methods Discussion of scientific advances based on relevant literature.

Results In genetics, technological advances allow for increases in speed and efficiency and decreases in costs in exploring the genetic underpinnings of disease processes, drug metabolism, treatment response, and survival. In patient-based research, advances yield empirically based and stringent approaches to measurement that are scientifically robust. Insights into the genetic basis of QOL will ultimately allow early identification of patients susceptible to QOL deficits and to target care. The Wilson and Cleary model for patient-reported outcomes was refined by incorporating the genetic underpinnings of QOL.

Conclusions This series of papers provides a path for QOL and genetics researchers to work together to move this field forward and to unravel the intricate interplay of the genetic underpinnings of patient-reported QOL outcomes. The ultimate result will be a greater understanding of the process relating disease, patient, and doctor that will have the potential to lead to improved survival, QOL, and health services delivery.

Keywords Quality of life · Patient-reported outcomes . Genetic variables · Theory · Clinical implications

\section{Introduction}

There is emerging evidence for a genetic basis of patientreported quality-of-life (QOL) outcomes that can be 
incorporated into clinical research and practice. In this paper, we provide arguments to indicate that scientific advances in genetics and patient-reported QOL research make this a timely endeavor. We further describe the clinical implications of this work and how this has the potential to expand and improve the quality of health care delivery. We also present a theoretical foundation for investigating the genetic underpinnings of patient-reported QOL, which delineates the hypothetical interconnections among the various components from the molecular level to clinical outcomes. Finally, we describe a series of papers resulting from the international and interdisciplinary GENEQOL Consortium that purports to investigate potential biological pathways, genes, and genetic variants involved in QOL.

\section{Scientific imperatives}

The fields of genetics and patient-reported QOL research have experienced significant advances in recent years. Genetic research has burgeoned despite the myriad of genetic variables. Technical advancements allow for increases in speed and efficiency and reduced costs of genetics research, such as high-throughput sequencing and new generations of micro-array techniques (see the Appendix that includes a glossary of genetic terms). The rapid expansion of the exploration of the human genome involving genome-wide association studies (GWAS) has made it possible to identify a large number of robust associations between specific chromosomal loci and complex human diseases, such as type 2 diabetes and rheumatoid arthritis [1]. Since 2007, GWAS have identified more than 250 genetic loci of common genetic variants involved in these and other common diseases. According to Hirschhorn [2; page 1699], this 'explosion represents one of the most prolific periods of discovery in human genetics'. Identifying genetic markers that are risk factors for disease and delineating the genetic underpinnings of disease processes, drug metabolism, treatment response, and survival have a central place on the research agenda. Initial explorations into gene-environment interactions have caused genetic researchers to think beyond a strictly molecular level to include interactions at the level of the organism and the world around the organism [3].

Before we describe the scientific advances of QOL research, we will first clarify the key components. Since the focus of this paper is on QOL, we will either refer to QOL when we mean it to be applicable to individuals in general, or to the newer term patient-reported (as synonymous to health-related) QOL when we mean the narrower concept applicable to patients. We will use the more general term patient-reported outcomes (PRO) when we refer to any report coming directly from patients [4].
Patient-based QOL research has progressed in parallel to genetics research. A number of patient-reported QOL measures are available that have the requisite measurement properties for routine use in clinical research. Clearly, PRO-related research differs from genetic research in the source of information. However, the two research fields have more in common than meets the eye. For example, both fields face challenges caused by multiplicity of outcomes, power considerations, missing data, and validation issues. It should also be noted that all measures are associated with error, whether they are obtained by self-report, physical examination, or in the laboratory [5]. Therefore, there is no inherent difference in variability and/or measurement error derived from PRO-based science than from test tube-based science. For example, it is well known but often ignored that there are multiple sources of variability and error in genotyping an individual $[6,7]$.

There are also detailed guidelines [8-10] and checklists [11] on how to conduct scientifically sound patient-reported QOL research and to circumvent previously identified barriers to implementation. More specifically, it is now well established that existing patient-reported QOL measures are as reliable as most other clinical outcomes, including clinician-rated outcomes of patient well-being and laboratory data [5]. This finding holds particularly at the group level. Furthermore, the guidelines that are available have proven to be useful: the quality of trialbased QOL data has improved over time [12]. Moreover, one of the most provocative findings in QOL research is that patient-reported assessments do not only have unique prognostic value but are in some cases even superior to clinician-based evaluations and biomedical assessments for predicting survival in general populations [13] and chronically ill patients [14-17]. Finally, clinical significance of patient-reported QOL data can be established, given the currently available guidelines [18-20]. In summary, these advances establish that patient-reported QOL outcomes, built on empirically based and stringent approaches to measurement, are scientifically robust and do not need to be viewed nor handled differently than any other parameter in medical research [21].

Investigations of the genetic basis of PROs are emerging. Studies of twins have indicated that heritability estimates for QOL-related domains such as mood and selfreported health range from 20 to 50\% [22-24]. This level of heritability is comparable or even higher than that of most diseases. For example, the heritability among the general population is $42 \%$ for prostate cancer, $35 \%$ for colorectal cancer, and $27 \%$ for breast cancer. Smaller percentages of heritability were found for cancer of the stomach, lung, pancreas, ovary, and bladder [25]. Biological pathways have been associated with PROs such as pain, fatigue, mood, and overall well-being. As a note of 
caution, we would like to highlight that despite high levels of heritability and known biological pathways, the search for the myriad of genes and gene interactions involved in QOL is far from straightforward. Nevertheless, a number of genes related to these pathways have been identified. The linkage between patient-reported QOL and genetic variables has begun to be explored and yields promising results. Sloan and Zhao [26] were the first to examine the direct link between polymorphisms and cancer patients' QOL, using a large randomized North Central Cancer Treatment Group clinical trial. More than triple the number of relationships between genetic variables and patient-reported QOL outcomes were observed than would be expected by chance alone. They found evidence for relationships between overall QOL, symptom distress, and fatigue with variant genotypes of three enzymes involved in folate metabolism. Recently, Yang et al. [27] evaluated the role of glutathionerelated genotypes on QOL in advanced non-small cell lung cancer patients who participated in a clinical trial. Patients carrying the glutathione peroxidase 1 (GPX1-CC) genotype had a clinically significant decline in overall QOL, and physical, functional, and emotional well-being. Clearly, these findings need to be replicated in future independent samples. However, the findings are sufficiently compelling to justify further studies to delineate the genes that are involved in patient-reported QOL and the extent to which they impact such PROs.

\section{Clinical implications}

The actual translation of genetic knowledge into clinical practice is one of the key challenges for the next decades [28, 29]. Genetic factors can be influenced by complex interactions with other genes, disease processes, and environmental factors that may change over time, making it difficult to know how to translate knowledge of these processes into clinical practice. Moreover, practical applications need to take issues of validity of the complex measurements into account [29].

Our vision for the future is nonetheless that emerging insight into the genetic basis of patient-reported QOL domains will ultimately allow us to explore new pathways for improving patient care. Knowledge of the biological pathways through which various genetic predispositions propel people toward negative or away from positive health experiences will ultimately transform health care. If we can identify patients who are susceptible to poor QOL, we will be able to better target preventive strategies and/or specific support, such as interventions inducing lifestyle and behavioural changes, psychological counseling or therapy, and/or pharmacological treatment. Such early interventions may have a profound impact enabling people to experience substantially greater well-being because they involve the underlying genetic biology of health (see Theoretical Underpinnings). Results of this work will thus enable health care providers to screen for patients who are likely to experience symptoms and QOL deficits from disease and its treatments. We hope that as a consequence, clinicians will be able to intervene prophylactically, monitor patient well-being, improve treatment decision-making, and improve outcomes encompassing survival, QOL, and satisfaction with care. Ultimately, QOL can then become an integral component in the genetic profile. To cite Sloan and Zhao [26], "Doctors will eventually use genetic patterns for several tasks: to tell whether a cancer will spread, to predict how various therapies such as specific drugs or radiation will work, and perhaps even to see how someone's QOL will be affected."

\section{Theoretical underpinnings}

The analysis of the genetic disposition of patient-reported QOL requires a model to delineate the hypothetical relationships among QOL domains, biological mechanisms, and genetic variants. Such a model would need to go beyond the evident relationships among biological factors and disease-related symptoms, to acknowledge that QOL also comprises overall physical, social, and psychological well-being. We have adopted the widely used theoretical model of Wilson and Cleary [30] that links biological factors and patient-reported QOL. We propose a number of refinements of this model by first including the genetic underpinnings of biological/physiological variables as well as of individual characteristics (see Fig. 1). The refinement also proposes a mutual influence between individual characteristics and genetic and biological factors, respectively. Additionally, the model allows for interactions to occur between environment and genetic/biological factors (e.g., epigenetic mechanisms). Further, the model refinement involves temporal relationships that may change over time, rendering the model explicitly dynamic. These and other refinements are described in more detail below. But first we will present the original model.

The model proposed by Wilson and Cleary [30] describes a continuum of interrelated levels/measures of health that can be ordered from more biological (left) to more psychological complexity and integration (right). Biological and physiological factors are related to the function of cells (e.g., creatinine levels), organs (e.g., pulmonary wheezes), and organ systems (e.g., the diagnosis of colorectal cancer). When the focus is shifted from cells or specific organ systems to the person as a whole, the first level encountered is symptom status. This category was defined as "the patient's perception of an abnormal 
Fig. 1 Extended model of Wilson and Cleary [29]. Interrupted arrows and words in italics are added to the original model. Bold arrows were original in standard font and highlight the increasingly acknowledged importance of the relationships they depict

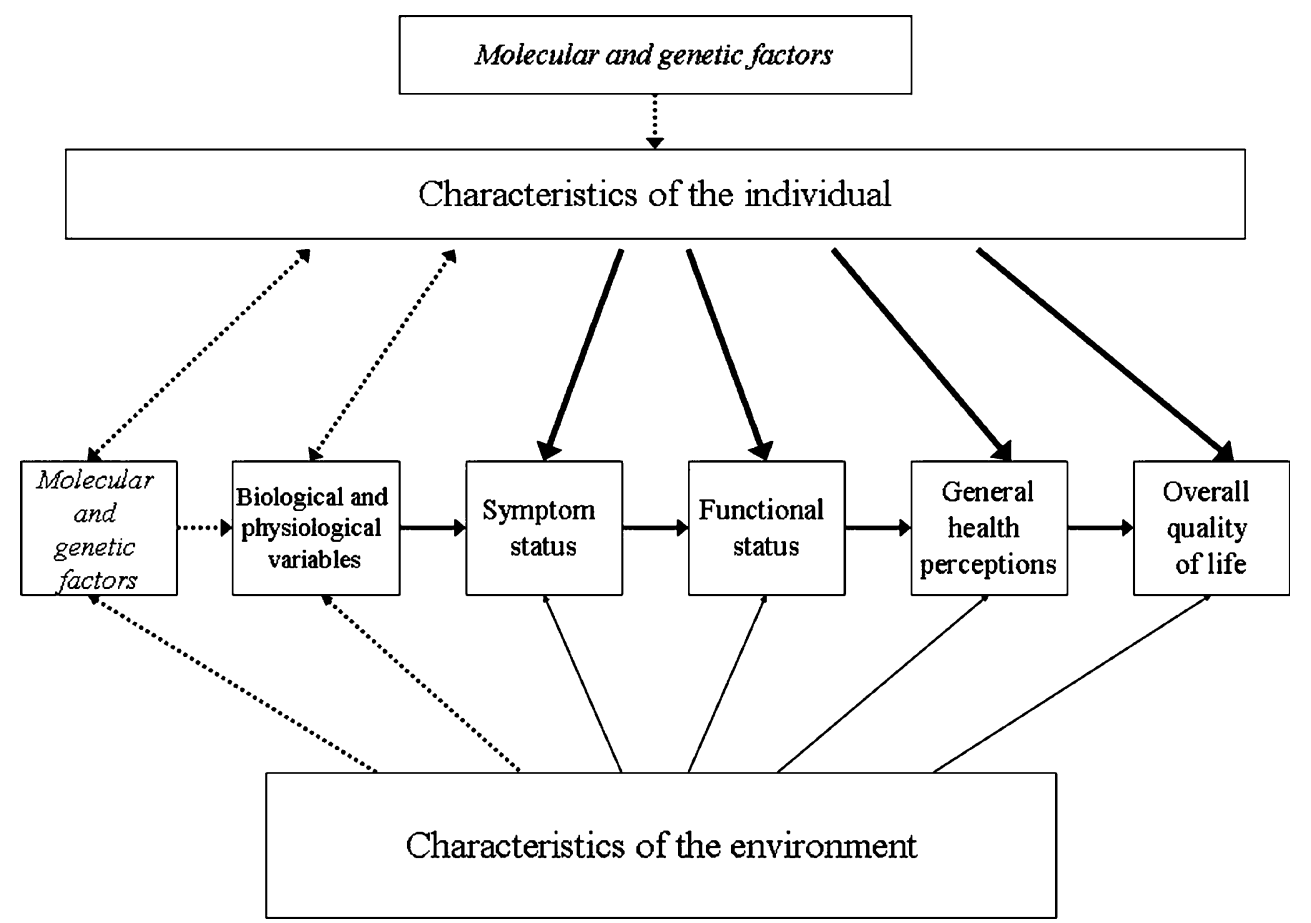

physical, emotional, or cognitive state" [30; page 61], e.g., knee pain or worry. Functional status comprises physical, social, role, and psychological behavior and is related to the ability to perform particular defined tasks, e.g., ability to walk up stairs or handling stressful situations. General health perceptions refer to subjective evaluations of physical as well as mental health. Overall quality of life pertains to subjective assessments of how happy or satisfied the individual is with his/her life in the context of a specific physiological state, symptom experience, functional status, and subjective state of physical and mental well-being.

The arrows in the model indicate causal relationships, such that biological and physiological variables affect symptom status, which in turn impacts functional status and so on. Additionally, characteristics of the individual and of the environment also exert an impact on the different health outcomes. The more one moves to the right, the larger this impact becomes. Wilson and Cleary [30] also pointed out that the arrows in the model depict the dominant associations, but that many reciprocal and other relationships exist.

We propose the following extensions of this model to accommodate emerging evidence of the genetic underpinning of QOL. First, whereas the level of molecular and genetic factors was implicit in the original model, we included it as a separate category at the far left. Wilson and Cleary [30; page 60] acknowledged that the most fundamental biological determinants of patient-reported QOL are molecular and genetic factors. However, these factors were not incorporated explicitly in their model because in 1995 (date of publication), these factors were not commonly measured and applied in routine clinical practice. The current abundance of genetic research addressing biological causes and courses of disease warrants this separate category in the model. These molecular and genetic factors thus impact symptom status, functional status, general health perceptions, and overall QOL indirectly via their involvement in the underlying disease.

Second, at the time Wilson and Cleary [30] published their model, the idea of a relationship between genes and QOL was far from evident. For example, the notion that QOL would be genetically determined had not appeared in the medical literature. Currently, there is mounting evidence that genes impact the experience of symptoms, perceptions of health, mood, and QOL in general. Therefore, we have also incorporated molecular and genetic factors as a separate category impacting characteristics of the individual. It follows that these genetic factors impact symptom status, functional status, general health perceptions, and overall QOL, via individual characteristics. These sets of genes may, in part, be the same genes involved in the underlying condition or disease process. For example, genes involved in the etiology or biology of pain may be similar to those involved in the subjective experience of pain. Other genes may be involved as well, particularly those that impact individual appraisals and experiences of health and life, e.g., via perception, personality, mood, and outlook on life.

Third, Wilson and Cleary [30] built their framework around dominant unidirectional relationships. We propose to add bidirectional arrows from characteristics of the individual to molecular and genetic factors, and biological 
and physiological variables, respectively. Clearly, our genetic and biological makeup has repeatedly been found to shape the way we are. In other words, the upward arrows are empirically warranted and beyond dispute. For a long time, clinicians have been convinced of the reverse impact of our minds on our body. A well-studied example is pain. There is emerging evidence that the expectation of clinical benefit is a major trigger for the placebo effect. Applied to pain, the expectation of pain relief in response to placebo analgesia may activate the reward circuitry and the release of dopamine in the ventral striatum, which in turn may trigger the endogenous opioid system [31]. A maladaptive cognitive and emotional response to pain is catastrophizing, which involves feelings of helplessness, rumination, and aggrandizing pain-related complaints. Catastrophizing has been found to be consistently associated with elevated experiences of pain, probably via its effects on the central nervous, neuromuscular, cardiovascular, immune, and neuroendocrine systems [32]. Although evidence of the impact of cognitive processes and psychological reactions on bodily processes at the molecular level is emerging, it has yet to be scientifically articulated. We included this relationship in the model to indicate that it is an intriguing new frontier. In the not too distant future, we will no doubt know whether this bidirectionality is empirically warranted.

Fourth, we added unidirectional arrows from environment to molecular and genetic factors and biological and physiological variables. There is emerging evidence that the environment can alter gene expression, by down- or upregulation of the synthesis of specific proteins that affect an individual's mental state, by epigenetic mechanisms. For example, research in rodents indicated that parental nurturing behavior can bolster emotional resilience in the recipient by boosting the expression of the gene that modulates anxiety. Conversely, distressing events can turn off the expression of a neuronal growth protein, thereby eliciting depression [33].

Fifth, we thickened the arrows leading from characteristics of the individual to symptom status, functional status, general health perceptions, and overall QOL, as a minor adaptation. In this way, the increasing evidence is highlighted that individual characteristics, such as personality, illness perceptions, expectations, values, and coping strategies, can exert larger effects on PROs than biological and physiological variables, such as the underlying disease.

The added arrows to the Wilson and Cleary [30] model signify the intricate interplay between individual, biological, and environmental characteristics at the most fundamental level. It should be noted that the current model is a necessary simplification as multiple determinants interact and operate in nested genetic, biological, behavioural, and environmental contexts that change as a person develops. Thus, the temporal relationships, while not explicitly addressed in the Wilson and Cleary model, may have a profound impact on each outcome [34]. Iterative interactions may occur over time (not depicted in the figure). For example, our genetic make up will influence our individual characteristics, such as personality and generalized tendencies to appraise experience (e.g., optimistic versus catastrophizing). This in turn will not only affect our overall psychological well-being but also our health and symptom experience, by shaping and perhaps transforming the underlying biological substrate. This in turn may affect an individual's psychosocial environment, and so on.

The suggested modifications cause the model to become symmetrical. Although it may be a mere graphical artifact, it is appealing because the gestalt it displays highlights the interplay between body and mind, or perhaps better, between brain and mind. It also demonstrates that QOL is not separate from biological factors. Rather, it represents the integration of all the preceding factors, including genetic, physiological, perceptual, and behavioral.

\section{Overview of papers}

The aim of the current series of papers is to highlight that the time has come to investigate the genetic basis of patient-reported QOL and to stimulate investigations into the novel and intriguing questions surrounding the genetic disposition of QOL. In recognition of the magnitude of this task, we have taken a first step in answering this call to action by establishing the GENEQOL Consortium.

In pursuing the delineation of the relationship between genes and QOL, both genetic and QOL research is hindered by a mono-disciplinary approach. Few genetic researchers use QOL endpoints, and similarly few QOL researchers are engaged in genetic research. Therefore, it is of paramount importance to join forces among the disparate disciplines. This primary aim of the international and interdisciplinary GENEQOL Consortium is to provide the requisite foundation and research culture to stimulate the development of this multi-disciplinary field of research. We started to identify five primary patient-reported QOL outcomes as initial targets: self-rated physical health, pain, fatigue, negative psychological affect, and positive psychological affect. The first tangible objective of the GENEQOL Consortium was to develop a list of potential biological pathways, genes, and genetic variants involved in these QOL outcomes, by reviewing genetic studies. The establishment of the Consortium and its first results has been described in an omnibus paper [35].

This special series of papers informs readers of Quality of Life Research about this groundbreaking and emerging area of research and provides a more detailed summary of its first results than was possible in the context of the 
omnibus paper. The series covers both the advances in genetic and patient-reported QOL research that allow for further investigation of the genetic underpinnings of such PROs, but also articulates outstanding questions. It starts with the current paper and is followed by a reflection from a patient perspective by Chauhan [36]. She emphasizes that researchers interested in the genetic disposition of QOL share with patients complementary goals on the journey to enhance patients' QOL. By developing scientific validation for patient-reported experiences, researchers help the clinicians to focus more on the patients' perceptions. Identification of the genetic components validates the reality of the patient experience and makes clinicians more amenable to that reality as an important component of disease progression and treatment plans.

Shi and colleagues [37] address pain and provide an overview of the current knowledge of pain- and analgesicrelated pathways, genes, and genetic variants. The authors describe three categories of potential genetic pathways for pain perception. They also present the genes related to pharmacodynamics (receptor interactions, intracellular signaling, and modulation of opioid effects) and pharmacokinetics (metabolism and transport) of responses to analgesics.

The paper by Barsevick and colleagues [38] addresses the biological and genetic mechanisms of cancer-related fatigue. This significant symptom affects over $60 \%$ of cancer patients. The authors first describe a number of pathophysiological pathways that are hypothesized to be involved in fatigue, including, cytokine dysregulation, hypothalamic-pituitary-adrenal axis dysfunction, neurotransmitter dysregulation, and disruption of the circadian rhythm. The authors also address current evidence for the genetic control of cancer-related fatigue. For example, a number of cytokine genes and their polymorphisms have been proposed as candidate markers for the study of cancer-related fatigue. The identification of these pathways and genetic control mechanisms hold promise for the identification of effective treatments.

The paper by Sprangers and colleagues [39] focus on individuals' emotional states. These authors provide a summary of the heritability studies on negative as well as positive emotional states and show that heritability estimates for anxiety and depression $(30 \%-40 \%)$ are slightly lower than those for positive emotional states (40\%-50\%). Additionally, biological pathways and genetic variants are described that are involved in these affect states. The hypothalamo-pituitary-adrenal axis was found to be a central system in the etiology of depression. Biological and genetic backgrounds of positive emotional states were found to be rarely investigated.

The work by the GENEQOL Consortium is expanded to include the investigation of relationships between genetic variables and QOL of over 8,000 mothers and pregnant women and near to 6,000 of their young children [40]. QOL of mothers and children was measured longitudinally and DNA was extracted. Candidate genes have been studied and GWAS of QOL of both mothers and young children will be performed. This study is an example of a large birth cohort study that is one of the first to provide insight into the genetic underpinning of QOL of mothers and their young children. Moreover, the authors provide a template for data analyses to stimulate further research.

\section{Parting thoughts: the path forward}

The major aim of this series of publications is to stimulate researchers in the fields of genetics and patient-reported QOL to become engaged in this broad-ranging scientific endeavor. We are well aware that the combination of two scientific fields does not only bring together formidable scientific expertise but also compounds the scientific challenges inherent to each field. We hope that this series of papers will provide a path for QOL and genetics researchers to join forces to move this field forward in tangible ways and to unravel the intricate interplay of the genetic underpinnings of patient-reported QOL outcomes. The ultimate result will be a better understanding of the process relating disease, patient, and doctor that will have the potential to lead to improved survival, QOL, and health services delivery.

Acknowledgments We are indebted to Carolyn Schwartz for her support, suggestion to adopt a theoretical model linking patientreported QOL with genetic variables, and stimulating discussions. We would also like to thank all members of the GENEQOL Consortium for their astute and helpful comments to the current draft, their invaluable contributions to the papers in this series and their contribution to the Consortium activities at large. We thank anonymous reviewers for helpful suggestions to further improve the text.

Open Access This article is distributed under the terms of the Creative Commons Attribution Noncommercial License which permits any noncommercial use, distribution, and reproduction in any medium, provided the original author(s) and source are credited.

\section{Appendix A: Glossary}

Chromosome: Self-replicating structures in the nucleus of a cell that carry the genetic information [41].

DNA (deoxyribonucleic acid): The double-stranded molecule that encodes genetic information [42].

Epigenetics: The study of heritable changes to DNA structure that do not alter the underlying sequence [43]. Gene: The basic unit of inheritance. A sequence of DNA that codes for a particular protein product [42].

Genome: The entire collection of genetic information (or genes) that an organism possesses [41]. 
Genome-wide association study (GWAS): A study that evaluates association of genetic variation with outcomes or traits of interest by using 100,000-1,000,000 markers or more across the genome [41].

Genotype: The genetic constitution of an individual [41]. Heritability: The proportion of phenotypic differences among individuals that can be attributed to genetic differences in a particular population [42].

High-throughput sequencing: High-speed and low-cost DNA sequencing.

Locus (plural, loci): The site(s) on a chromosome at which the gene for a particular trait is located [41].

Phenotype: An observed characteristic of an individual that results from the combined effects of genotype and environment [42].

Polymorphism: The existence of two or more variants of a gene, occurring in a population, with at least $1 \%$ frequency of the less common variant (cf mutation) [41]. Twin study: Study comparing the resemblance of identical and fraternal twins to estimate genetic and environmental components of variance [42].

\section{Appendix B: GENEQOL consortium participants per December 2009}

Amy P. Abernethy, Duke Cancer Care Research Program, Duke University Medical Center, Durham, NC, US; Frank Baas, Laboratory of Neurogenetics, Academic Medical Center, University of Amsterdam, Amsterdam, The Netherlands; Andrea M. Barsevick, Cancer Prevention and Control Program, Fox Chase Cancer Center, Philadelphia, PA, US; Meike Bartels, Department of Biological Psychology, VU University, Amsterdam, the Netherlands; Dorret I. Boomsma, Department of Biological Psychology, VU University, Amsterdam, the Netherlands; Andrew Bottomley, Quality of Life Department, EORTC Data Center, Brussels, Belgium; Michael Brundage, Department of Oncology, Queen's University Cancer Centre of Southeastern Ontario, Kingston, Ontario, Canada; Cynthia Chauhan, Cancer Advocacy, Wichita, KS, US; David Cella, Department of Medical Social Sciences, Feinberg School of Medicine, Chicago, IL, US; Charles S. Cleeland, Department of Symptom Research, The University of Texas M. D. Anderson Cancer Center, Houston, TX, US; Corneel Coens, Quality of Life Department, EORTC Data Center, Brussels, Belgium; Amylou C. Dueck, Section of Biostatistics, Mayo Clinic, Scottsdale, AZ, US; Marlene H. Frost, Women's Cancer Program, Mayo Clinic, Rochester, MN, US; Per Hall, Department of Medical Epidemiology and Biostatistics, Karolinska Institute, Stockholm, Sweden; Michele Y. Halyard, Department of Radiation Oncology, Mayo Clinic,
Scottsdale, AZ, US; Pål Klepstad, Department of Intensive Care Medicine, St Olavs University Hospital, Norwegian University of Technology and Science, Trondheim, Norway; Nicholas G. Martin, Queensland Institute of Medical Research, Brisbane, Australia; Christine Miaskowski, School of Nursing, University of California, San Francisco, CA, US; Miriam Mosing, Queensland Institute of Medical Research, Brisbane, Australia; Benjamin Movsas, Department of Radiation Oncology, Henry Ford Health System, Detroit, MI, US; Joao R. Oliveira, Department of Neuropsychiatry, Federal University of Pernambuco, RecifePernambuco, Brazil; Juan Ordoñana, Department of Human Anatomy and Psychobiology, University of Murcia, Murcia, Spain; Donald L. Patrick, Department of Health Services, University of Washington, Seattle, WA, US; Nancy L. Pedersen, Department of Medical Epidemiology and Biostatistics, Karolinska; Institute, Stockholm, Sweden; Hein Raat, Preventive Youth Health Care, Erasmus Medical Center, Rotterdam, the Netherlands; Bryce Reeve, Division of Cancer Control and Population Sciences, National Cancer Institute, Bethesda, MD, US; Mary E. Ropka, Cancer Prevention and Control Program, Fox Chase Cancer Center, Cheltenham, PA, US; Quiling Shi, Department of Symptom Research, The University of Texas M. D. Anderson Cancer Center, Houston, TX, US; Gen Shinozaki, Department of Psychiatry and Psychology, Mayo Clinic, Rochester, MN, US; Jasvinder A. Singh, Minneapolis Veterans Affairs Medical Center and University of Minnesota, Minneapolis, $\mathrm{MN}$ and Mayo Clinic College of Medicine, Rochester, MN, US; Jeff A. Sloan, Department of Health Sciences Research, Mayo Clinic, Rochester, MN, US; Mirjam A. G. Sprangers, Department of Medical Psychology, Academic Medical Center, University of Amsterdam, Amsterdam, The Netherlands; Dick Swaab, The Netherlands Institutes for Neuroscience, Amsterdam, the Netherlands; Cornelis J. F. Van Noorden, Department of Cell Biology and Histology, Academic Medical Center, University of Amsterdam, Amsterdam, The Netherlands; Ruut Veenhoven, Faculty of Social Sciences, Erasmus University Rotterdam, Rotterdam, The Netherlands; Gert Wagner, Berlin University of Technology, Max Planck Research School LIFE, Berlin, Germany; Ping Yang, Department of Genetic Epidemiology, Mayo Clinic, Rochester, MN, US; Ailko H. Zwinderman, Department of Clinical Epidemiology and Biostatistics, Academic Medical Center, University of Amsterdam, Amsterdam, The Netherlands.

\section{References}

1. Hardy, J., \& Singleton, A. (2009). Genomewide association studies and human disease. New England Journal of Medicine, $360,1759-1768$. 
2. Hirschhorn, J. N. (2009). Genomewide association studiesIlluminating biologic pathways. New England Journal of Medicine, 360, 1699-1701.

3. Seabrook, J. A., \& Avison, W. R. (2010). Genotype-environment interaction and sociology: Contributions and complexities. Social Science and Medicine, 70, 1277-1284.

4. Patrick, D. L., Burke, L. B., Powers, J. H., Scott, J. A., Rock, E. P., Dawisha, S., et al. (2007). Patient-reported outcomes to support medical product labeling claims: FDA perspective. Value Health, 10, S125-S137.

5. Hahn, E. A., Cella, D., Chassany, O., Fairclough, D. L., Wong, G. Y., Hays, R. D., et al. (2007). Precision of health-related qualityof-life data compared with other clinical measures. Mayo Clinic Proceedings, 82, 1244-1254.

6. Hüebner, C., Petermann, I., Browning, B. L., Shelling, A. N., \& Ferguson, L. R. (2007). Triallelic single nucleotide polymorphisms and genotyping error in genetic epidemiology studies: MDR1 (ABCB1) G2677/T/A as an example. Cancer Epidemiology, Biomarkers and Prevention, 16, 1185.

7. Pluzhnikov, A., Below, J. E., Konkashbaev, A., Tikhomirov, A., Kistner-Griffin, E., Roe, C. A., et al. (2010). Spoiling the whole bunch: Quality control aimed at preserving the integrity of highthroughput genotyping. The American Journal of Human Genetics, 87, 123-128.

8. Fayers, P. M., Hopwood, P., Harvey, A., Girling, D. J., Machin, D., \& Stephens, R. (1997). Quality of life assessment in clinical trials-guidelines and a checklist for protocol writers: The UK Medical Research Council experience. MRC Cancer Trials Office. European Journal of Cancer, 33, 20-28.

9. De Haes, J., Curran, D., Young, T., Bottomley, A., Flechtner, H., Aaronson, N., et al. (2000). Quality of life evaluation in oncological clinical trials- the EORTC model. European Journal of Cancer, 36, 821-825.

10. Bottomley, A., Jones, D., \& Claassens, L. (2009). Patientreported outcomes: Assessment and current perspectives of the guidelines of the Food and Drug Administration and the reflection paper of the European Medicines Agency. European Journal of Cancer, 45, 347-353.

11. Efficace, F., Bottomley, A., Osoba, D., Gotay, C., Flechtner, H., D'haese, S., et al. (2003). Beyond the development of healthrelated quality of life (HRQOL) measures. A checklist for evaluating HRQOL outcomes in cancer clinical trials-does HRQOL evaluation in prostate cancer research inform clinical decisionmaking? Journal of Clinical Oncology, 21, 3502-3511.

12. Efficace, F., Osoba, D., Gotay, C., Sprangers, M., Coens, C., \& Bottomley, A. (2007). Has the health-related quality of life reporting in clinical cancer trials improved over time? Towards bridging the gap with clinical decision making. Annals of Oncology, 18, 775-781.

13. Idler, E. L., \& Benyamini, Y. (1997). Self-rated health and mortality: A review of 27 community studies. Journal of Health and Social Behavior, 38, 21-37.

14. Singh, J. A., Nelson, D. B., Fink, H. A., \& Nichol, K. L. (2005). Health-related quality of life predicts future health-care utilization and mortality in veterans with self-reported physician-diagnosed arthritis: The veterans arthritis quality of life study. Seminars in Arthritis Rheumatism, 34, 755-765.

15. Cunningham, W. E., Crystal, S., Bozzette, S., \& Hays, R. D. (2005). The association of health-related quality of life with survival among persons with HIV infection in the United States. Journal of General Internal Medicine, 20, 21-27.

16. Mapes, D. L., Lopes, A. A., Satayathum, S., Mccullough, K. P., Goodkin, D. A., Locatelli, F., et al. (2003). Health-related quality of life as a predictor of mortality and hospitalization: The Dialysis Outcomes and Practice Patterns Study (DOPPS). Kidney International, 64, 339-349.
17. Gotay, C. C., Kawamoto, C. T., Bottomley, A., \& Efficace, F. (2008). The prognostic significance of patient-reported outcomes in cancer clinical trials. Journal of Clinical Oncology, 26, $1355-1363$

18. Revicki, D., Hays, R. D., Cella, D., \& Sloan, J. (2008). Recommended methods for determining responsiveness and minimally important differences for patient-reported outcomes. Journal of Clinical Epidemiology, 61, 102-109.

19. Norman, G. R., Wyrwich, K. W., \& Patrick, D. W. (2007). The mathematical relationship among different forms of responsiveness coefficients. Quality of Life Research, 16, 815-822.

20. Crosby, R. D., Kolotkin, R. L., \& Williams, G. R. (2003). Defining clinically meaningful change in health-related quality of life. Journal of Clinical Epidemiology, 56, 395-407.

21. Sprangers, M. A. G. (2010). Disregarding clinical trial-based patient-reported outcomes is unwarranted: Five advances to substantiate the scientific stringency of quality-of-life measurement. Acta Oncologica, 49, 155-163.

22. Nes, R. B., Røysamb, E., Tambs, K., Harris, J. R., \& ReichbornKjennerud, T. (2006). Subjective well-being: Genetic and environmental contributions to stability and change. Psychological Medicine, 36, 1033-1042.

23. Svedberg, P., Gatz, M., Lichtenstein, P., Sandin, S., \& Pedersen, N. L. (2005). Self-rated health in a longitudinal perspective: A 9-year follow-up twin study. Journal of Gerontology Series B: Psychological Sciences and Social Sciences, 60, S331-S340.

24. Romeis, J. C., Heath, A. C., Xian, H., Eisen, S. A., Scherrer, J. F., Pedersen, N. L., et al. (2005). Heritability of SF-36 among middle-age, middle-class, male-male twins. Medical Care, 43, $1147-1154$

25. Lichtenstein, P., Holm, N. V., Verkasalo, P. K., Iliadou, A., Kaprio, J., Koskenvuo, M., et al. (2000). Environmental and heritable factors in the causation of cancer. The New England Journal of Medicine, 343, 78-85.

26. Sloan, J., \& Zhao, X. (2006). Genetics and quality of life. Current Problems in Cancer, 30, 255-260.

27. Yang, P., Mandrekar, S. J., Hillman, S. H., Allen Ziegler, K. L., Sun, Z., Wampfler, J. A., et al. (2009). Evaluation of glutathione metabolic genes on outcomes in advanced non-small cell lung cancer patients after initial treatment with platinum-based chemotherapy: An NCCTG-97-24-51 based study. Journal of Thoracic Oncology, 4, 479-485.

28. Janssens, A. C., \& Van Duijn, C. M. (2009). Genome-based prediction of common diseases: Methodological considerations for future research. Genome Medicine, 18, 20.

29. Ioannidis, J. P. A. (2010). Genetics, personalized medicine, and clinical epidemiology: Expectations, validity, and reality in omics. Journal of Clinical Epidemiology, 63, 945-949.

30. Wilson, I. B., \& Cleary, P. D. (1995). Linking clinical variables with health-related quality of life. A conceptual model of patient outcomes. Journal of the American Medical Association, 273, $59-65$.

31. de la Fuente-Fernandez, R. (2009). The placebo-reward hypothesis: Dopamine and the placebo effect. Parkinsonism and Related Disorders, 15S3, S72-S74.

32. Campbell, C. M., \& Edwards, R. R. (2009). Mind-body interactions in pain: The neurophysiology of anxious and catatstrophic pain-related thoughts. Translational Research, 153, 97-101.

33. Higgins, E. S. (2009). The new genetics of mental illness. Scientific American Mind, 19, 41-47.

34. Halfon, N., \& Hochstein, M. (2002). Life course health development: An integrated framework for developing health, policy, and research. The Milbank Quarterly, 80, 433-479.

35. Sprangers, M. A. G., Sloan, J. A., Veenhoven, R., Cleeland, C. S., Halyard, M. Y., Abernethy, A. P., et al. (2009). The establishment of the GENEQOL Consortium to investigate the genetic 
disposition of patient-reported quality-of-life outcomes. Twin Research Human Genetics, 12, 301-311.

36. Chauhan, C. I am the quintessential optimist, I think that I have the quality-of-life gene in spades: reflections from a patient perspective (Commentary). Quality of Life Research, Epub ahead of print.

37. Shi, Q., Cleeland, C., Miaskowski, C., Klepstad, P., Pedersen, N., $\&$ The GENEQOL Consortium. Biological pathways and genetic variables involved in pain. Quality of Life Research, manuscript accepted for publication.

38. Barsevick, A., Frost, M., Zwinderman, A., Hall, P., Halyard, M. I., \& The GENEQOL Consortium. I am so tired: Biological and genetic mechanisms of cancer-related fatigue. Quality of Life Research, manuscript accepted for publication.

39. Sprangers, M. A. G., Bartels, M., Veenhoven, R., Baas, F., Martin, N., Movsas, B., et al. Which patient will feel down, which will be happy? The need to study the genetic disposition of emotional states. Quality of Life Research, Epub ahead of print.
40. Raat, H., Van Rossem, L., Jaddoe, V. W. V., Landgraf, J. M., Feeny, D., Hofman, A., \& Mackenbach, J. P. Candidate gene study and genome-wide association study on health-related quality of life of mothers and young children: the Generation $\mathrm{R}$ study. Quality of Life Research, manuscript accepted for publication.

41. Attia, J., Ionannidis, J. P. A., Thakkinstian, A., McEvoy, M., Scott, R. J., Minelli, C., et al. (2009). How to use an article about genetic association A: Backgound concepts. Journal of the American Medical Association, 301, 74-81.

42. Plomin, R., DeFries, J. C., McClearn, G. E., \& McGuffin, P. (2008). Behavioral genetics (5th ed., pp. 1-532). New York: Worth Publishers and W. H. Freeman and Company.

43. Hardy, J., \& Singleton, A. (2009). Genomewide association studies and human disease. The New England Journal of Medicine, 360, 1759-1768. 